\title{
EFFICIENT MULTIMEDIA DELIVERY OVER DIGITAL SUBSCRIBER LINE
}

\author{
Haitao Zheng and K. J. Ray Liu \\ Department of Electrical Engineering and Institute for Systems Research \\ University of Maryland, College Park, MD 20742 \\ Tel: 301-405-6619, e-mail: haitaoz, kjrliu@eng.umd.edu
}

\begin{abstract}
We consider the design of a multimedia transmission systems to provide reliable and efficient delivery of multimedia data over digital subscriber line. The layers associated with different bit error performances are transmitted in parallel and the property of phone line channel is utilized to achieve unequal error protection efficiently. The transmitted power for the specified error performance is significantly reduced compared to the conventional schemes. Simulation results are provided for subband coded image and integrated services(video,voice and data), demonstrating excellent performance improvement, where a $0.5-2 \mathrm{~dB}$ average power gain is achieved.
\end{abstract}

\section{INTRODUCTION}

Asymmetric digital subscriber line(ADSL)[1], a transmission system capable of delivering very high bit rates over ordinary phone lines, has received tremendous interests recently. The wide variations in telephone line channel leads to increased difficulty and complexity for any single-carrier system. Multicarrier modulation (MCM) [2] is currently considered as a standard channel coding scheme for ADSL. A crucial aspect in the design of MCM system is the need to optimize the system transmission bandwidth and power through an optimal loading algorithm. The loading algorithms in literature can be divided into two categories. Category one computes bit rate and power distribution for given bit error rate(BER) $[2,3,4,5]$. Category two is based on minimizing the overall BER while reaching the data throughput under a power constraint. The overall BER is unknown until the final stage of optimization[6]. Multimedia data is often decomposed into hierarchical perceptually relevant layers, each of which has different perceptual importance. To deliver layered coded multimedia data through ADSL using the above loading algorithms, the layers of different importance are transmitted consecutively with the same BER, resulting in equal error protection. Unequal error protection can be achieved by varying the frame power during the transmission.

Recently, we have proposed a parallel transmission framework to provide unequal error protection efficiently for layered data transmission[7], which achieves significant performance improvement compared to the conventional loading algorithms. The layers are transmitted in parallel, each occupying a set of subchannels, as illustrated in Figure 1(a). The loading algorithm developed aims 
to minimize the channel induced distortion, under a given power and bit rate constraint. Therefore, it falls into category two. For the serial transmission that transmits the layers consecutively, the performance bottleneck is the huge power consumption for the layers with very high importance, thus very low $B E R$. The parallel transmission has the advantage that the very important layers are transmitted through the high channel gain to noise ratio(CGNR) subchannels, or good subchannels, as such, power consumption can be significantly reduced.

For some applications, the customers can choose the quality of the layers with flexibility. Therefore the importance of a layer can not be represented as the average distortion by a bit error as in [7], but according to customers' request. In particular, the layer's BER performance are specified by customers, hence the objective becomes how to satisfy customers' request while using the minimum amount of channel resources. Such consideration would help to increase the number of users that the system can support. In this paper, we develop a loading algorithm which handles the power and rate distribution for specified BER distribution, based on the parallel transmission framework proposed in [7]. The loading algorithm intends to minimize the overall transmitted power under a given bit rate constraint or, maximize the bit rate under a given power constraint.

\section{THE POWER OPTIMIZATION FOR GIVEN BERS}

We aim to develop a loading algorithm that achieves the specified BERs for the layers by distributing the transmitted power and bit rate among the subchannels. We assume that the layers are ordered in increasing BER order(decreasing importance order), and the subchannels are indexed in decreasing CGNR order. The optimization can be divided into two steps:

1. The Optimization Inside a Layer: Since the transmission time of the layers are fixed to be the same, for a given data throughput $B_{T}$, the number of bits from layer $i$ to be transmitted at a single frame is given by $B_{i}=\frac{B_{i t} t_{i} x}{B_{i t} t_{0} a}$, where $B i t_{i}$ represents the total bits of layer $i$ and Bit total represents the total bits of all the layers. Define the number of subchannels transmitting layer $n$ as $C_{n}, n=1 \ldots N$. If $\left\{C_{n}\right\}_{n=1}^{N}$ is given, the channel gain and noise variance of the subchannels corresponding to each layer is decided, and the power and bit rate optimization among the subchannels transmitting one particular layer can be performed independently to the other layers. The independence is also due to the knowledge of BER performance. For layer $i, i=1 \ldots N$, the loading algorithm for data transmission can be applied to equalize error performance to achieve $B E R_{i}$ and data throughput $B_{i}$. Figure $1(\mathrm{~b})$ shows the BER performances for the subchamels.

2. The Optimization Among the Layers: The optimization among the layers thus decides the distribution of $\left\{C_{n}\right\}_{n=1}^{N}$, with a constraint $\sum_{n=1}^{N} C_{n} \leq$ $C_{T}$, where $C_{T}$ is the number of subchannels in the MCM system. 


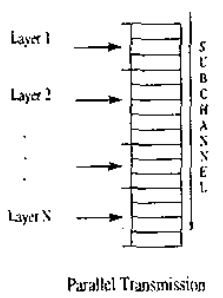

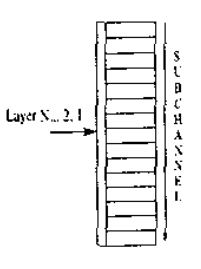

(a)

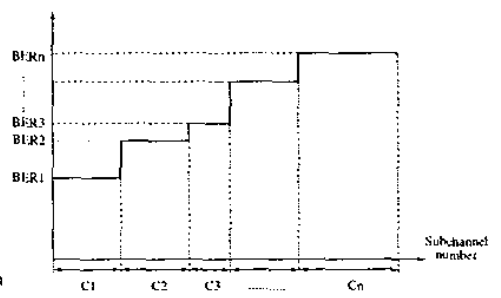

(b)

Figure 1: (a) Transmission Systems (b)BER Distribution for the subchannels

\section{Bit and Power Loading for Given Number of Subchannels}

For each layer, if the number of subchannels occupied is decided, the conventional loading algorithm can be used to allocate the power and bit rate among the subchannels transmitting the same layer. On an AWGN channel, with input energy $E_{s}$, CGNR $G$, the maximum data rate or capacity is $C=\log _{2}\left(1+E_{s} G\right)$ per herz. Any reliable and implementable system has to transmit a data rate below such capacity. For a given symbol error probability $P e$ and rate $b$, an SNR gap is $\Gamma=\frac{2^{c}-1}{2^{b}-1}=\frac{S N R}{2^{b}-1}$ where $S N R=E_{s} G$. For uncoded QAM, Pe $=10^{-6}$ corresponds to a constant $\Gamma=8.8 d B$. Equivalently, the rate $R$ can be expressed as a function of $S N R$ and $\Gamma, b=\log _{2}\left(1+\frac{S N R}{\Gamma}\right)$. The goal is to minimize the overall transmitted power $E$, for a set of $M$ parallel subchannels with throughput of $R$ at a specific SNR gap $\Gamma$. For subchannel $m$, if using QAM, the transmitted power can be expressed via the gap approximation as $E_{m}\left(b_{m}\right)=\frac{\Gamma}{g_{m}}\left(2^{b_{m}}-1\right)$, where $b_{m}$ and $g_{m}$ are the bit rate and CGNR of subchannel $m$, respectively. The loading algorithm can start from any arbitrary $\left\{b_{m}\right\}_{m=1}^{M}$ distributions, and approach the optimal solution successively. To maximize the overall bit rate under a given power constraint, the same algorithm can be applied. Further information about this algorithm can be found in [3].

\section{Number of Subchannels Allocation}

Since the number of subchannels used for all the layers is limited to $C_{T}$, the optimization can be achieved by integer programming. In practice, the number of modulator/demodulators at the transmitter and receiver is limited to $\left[R_{\min }, R_{\max }\right]$. For QAM, if $\left[R_{\min }, R_{\max }\right]=[2,6]$, then the allowed modulations are QAM4, QAM8, QAM16, QAM32, QAM64. The number of subchannels that each layer can use has a upper and lower bound as:

$$
C_{n, \min }=\frac{B_{n}}{R_{\max }}, \quad C_{n, \max }=\frac{B_{n}}{R_{\min }} .
$$

Assigning $\left\{C_{n}=C_{n, m i n}\right\}_{n=1}^{N}$ is equivalent to transmit the data at the highest bit rate. Since the subchannels are ordered in a decreasing CGNR fashion, 
such assignment results in the minimum number of subchannel usage in the best CGNR region. However, the highest bit rate leads to a huge transmitted power consumption in order to achieve the specified BER. On the other hand, $\left\{C_{n}=C_{n, \max }\right\}_{n=1}^{N}$ intends to use the subchannel as much as possible so that the bit rate at each subchannel can be reduced. But at the same time, the transmitted power at some subchannels with very low CGNR will become the performance bottleneck. Observing the performance in the above two extreme cases, it can be concluded that the optimal solution is in between. Therefore, we propose a successive search method: starting from $\left\{C_{n}=C_{n, \min }\right\}_{n=1}^{N}$ and increase the number of subchannel occupied successively. At each searching stage, find the layer that yields the maximum decrease in the transmitted power by extending its subchannel by one. If adding an extra subchannel cannot reduce the power, stop the search. The detailed algorithm is described in [8].

\section{SIMULATION RESULT}

This section will present the simulations carried out on some typical layered source data. The performances of both the parallel transmission and the serial transmission are illustrated for comparison. The data throughput and source input are designed to be identical for the two transmission schemes for a fair comparison.

\section{Subband Coded Image}

We apply the proposed loading algorithm to transmit subband coded image over ADSL. Each subband is classified as a layer. The number of subchannels allowed in $\mathrm{MCM}$ is $C_{T}=256$. The bit rate is limited to the range $R_{\min }=2$ and $R_{m a x}=6$. For simplicity, we assume that subband 1 has BER1, subband 2 and 3 with BER2 and the rest with BER3. For serial transmission, the loading algorithm described in section is to achieve consistent BER performance among all the subchannels, where the frame powers during each layer transmission are computed corresponding to the layer's BER or $\Gamma$. Figure 2 plots the performance comparison as a function of data throughput $B_{T}$ at different source rates. Parallel transmission achieves as much as $2.5 \mathrm{~dB}$ PSNR improvement or $2 \mathrm{~dB}$ gain in terms of averaged transmitted power. Received image results are shown in Figure 3 .

\section{Integrated Service of Video, Voice and Data}

Wireline transmission systems will have to meet the needs of transmitting a variety of difference services, such as video, voice and data. Those services are associated with different data rate and different QoS requirement(in terms of BER). Therefore, each of them corresponds to a layer. Some typical examples are shown in Table 1. Therefore, they can be transmitted in parallel. We assume that each service is accompanied by an efficient rate control scheme to achieve a constant bit rate. The simulation results for $R_{\max }=6$ and $R_{\max }=$ 
12 are shown in Figure 4(a) which plots the averaged power consumption at different data throughput and different service requirement for $R_{\max }=6$. The performance for service requirement L3 at $R_{m a x}=12$ is also illustrated to show that increasing $R_{\max }$ will reduce the power consumption since it provides more options in the optimization. Figure $4(\mathrm{~b})$ also illustrates the performance at $R_{\max }=12$ in addition to the performance for $\mathrm{L} 1$ at $R_{\max }=6$. For L1, the extra 6 modulation selections lead to nearly $2 \mathrm{~dB}$ power gain. Overall, parallel transmission outperforms serial transmission.

Table 1. BER Distributions for Different Requirements

\begin{tabular}{|c|c|c|c|}
\hline Requirement & $\begin{array}{c}\text { Service } 1 \\
200 \mathrm{~K} \text { bits } / \mathrm{s}\end{array}$ & $\begin{array}{c}\text { Service } 2 \\
64 \mathrm{~K} \text { bits/s }\end{array}$ & $\begin{array}{c}\text { Service } 3 \\
10 \mathrm{~K} \text { bits } / \mathrm{s}\end{array}$ \\
\hline L1 & $10^{-6}$ & $10^{-5}$ & $10^{-3}$ \\
\hline L2 & $10^{-5}$ & $10^{-3}$ & $10^{-6}$ \\
\hline L3 & $10^{-3}$ & $10^{-5}$ & $10^{-6}$ \\
\hline
\end{tabular}

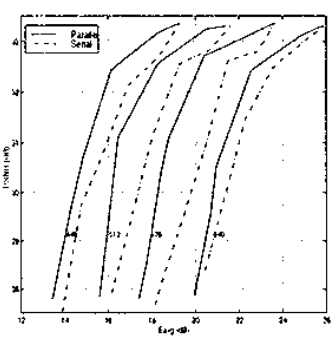

(a)

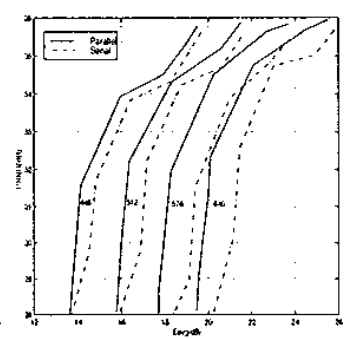

(b)

Figure 2: PSNR vs. Average Power at (a)0.5bpp and (b)1.0bpp Source Rate

\section{CONCLUSION}

We unveiled a new transmission system for layered coded data through ADSL. It aims to minimize the total transmitted power for a specified BER performances for different layers under a data throughput constraint. The layers with different QoS requirement(in terms of BER) are transmitted in parallel. This allows the data with higher priority to be transmitted through good subchannels as to provide unequal error protection naturally. The proposed algorithm shows $0.5-2 \mathrm{~dB}$ gain in terms of the averaged power per subchannel, compared to the conventional loading algorithms.

\section{References}

[1] K. Maxwell, "Asymmetric digital subscriber line: interim technology for the next forty years",IEEE Communication Mag.,pp.100-106, Oct 1996.

[2] J.A.C. Bingham, "Multicarrier modulation for data transmission:An idea whose time has come", IEEE Communication Mag.,May 1990. 
[3] J. Campello de Souza, "Optimal discrete bit loading for multicarrier modulation systems," ISIT,Boston, 1998.

[4] P.S. Chow, J.M. Cioffi and J.A.C. Bingham, "A practical discrete multiton transceiver loading algorithm for data transmission over spectrally shaped channels",IEEE Trans. on Comm.,pp. 773-775, Feb/March/April 1995.

[5] B.S. Krongold, K. Ramchandran and D.L. Jones, "Computationally Efficient Optimal Power Allocation Algorithm for Multicarrier Communication Systems", Proc. of ICC98, Atlanta, GA, June 1998.

[6] R.F.H. Fisher and J.B. Huber, "A new loading algorithm for discrete multiton transmission",Proc. of GlobalCOM 96, pp 724-728.

[7] H. Zheng and K.J.R. Liu, "Robust Image and Video Transmission over Spectrally Shaped Channels Using Multicarrier Moduation",IEEE Trans. on Multimedia, Vol. 1, No. 1, pp.88-103, March 1999.

[8] H. Zheng and K.J.R. Liu, "Power Minimization for Layer Coded Multimedia Data Transmission Over Digital Subscriber Line", submitted to JSAC, May 1999.

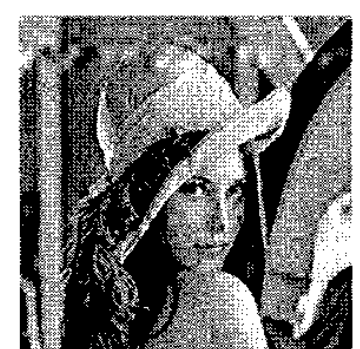

(a)

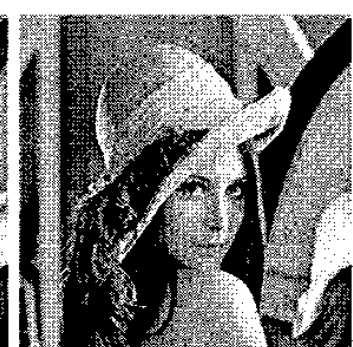

(b)

Figure 3: Received Image for $E_{a v g}=17 d B$ and $B_{T}=512$ of (a) serial and (b)parallel transmission

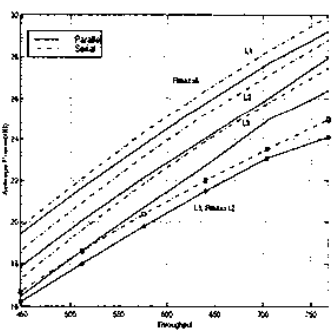

(a)

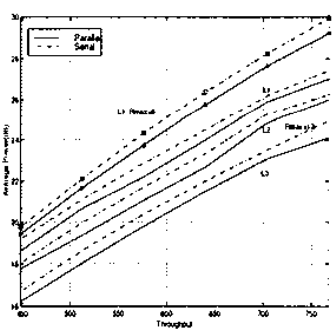

(b)

Figure 4: Transmitted Power vs. Throughput for (a) $R_{\max }=6$ and (b) $R_{\max }=8$ 